\title{
Pure laparoscopic liver resection versus percutaneous radiofrequency ablation for small hepatocellular carcinoma: a propensity score and multivariate analysis
}

\author{
Kai-Chi Cheng^, Kit-Man Ho \\ Department of Surgery, Kwong Wah Hospital, Hong Kong, China \\ Contributions: (I) Conception and design: KC Cheng; (II) Administrative support: KM Ho; (III) Provision of study materials or patients: KC Cheng; \\ (IV) Collection and assembly of data: KC Cheng; (V) Data analysis and interpretation: KC Cheng; (VI) Manuscript writing: Both authors; (VII) Final \\ approval of manuscript: Both authors. \\ Correspondence to: Dr. Kai-Chi Cheng. Department of Surgery, Kwong Wah Hospital, 25, Waterloo Road, Kowloon, Hong Kong, China. \\ Email: thomascheng@hotmail.com.
}

Background: In treatment of hepatocellular carcinoma (HCC), both laparoscopic liver resection (LLR) and radiofrequency ablation (RFA) provided similar short-term advantages. However, there was no robust clinical trial comparing the efficacy of LLR and RFA especially for small HCC. This study aimed to compare the short-term and long-term outcomes of LLR and RFA for patients with small HCC using a propensity score matching analysis to minimize potential selection bias. Factors affecting survival were then identified with multivariate analysis.

Methods: All patients underwent RFA or LLR for small HCC [defined as Barcelona Clinic Liver Cancer (BCLC) stage 0 or A, size $\leq 3 \mathrm{~cm}, \leq 3$ nodules on contrast CT scan or MRI with no evidence of macrovascular invasion] from April 2005 to August 2020 were included. Propensity score matching was conducted to match patients in the LLR group and RFA group. Prognostic indicators, i.e., age, gender, tumor size, tumor number, Child's grading, albumin, bilirubin, platelet count, international normalized ratio, alpha-fetoprotein level and presence of cirrhosis on imaging were chosen for propensity score calculation. The demographic data, tumor characteristics, operative data, post-operative outcomes and survival data of the two groups were compared. A multivariate analysis based on Cox regression was used to identify factors associated with survival.

Results: Median follow-up was 34 months. LLR and RFA had similar overall survival (91.8\% vs. $79.2 \%$ at 5-year, $\mathrm{P}=0.060)$; while the LLR had a significantly better disease-free survival (49.0\% vs. 30.3\% at 5-year, $\mathrm{P}=0.002)$ and local recurrence-free survival $(96.0 \%$ vs. $63.7 \%$ at 5 -year, $\mathrm{P}<0.001)$ when compared with the RFA. Multivariate analysis showed that treatment received by patient (LLR vs. RFA), prothrombin time and platelet counts were significantly associated with disease-free survival. On the other hand, the only factor associated with local recurrence-free survival was the treatment received by patient.

Conclusions: Both RFA and LLR are safe and feasible treatment options for patients with small HCC. LLR should be considered for patients with preserved liver function with a better disease-free survival; while RFA offered a comparable overall survival with less surgical trauma and shorter hospital stay.

Keywords: Hepatocellular carcinoma (HCC); laparoscopic hepatectomy; radiofrequency ablation (RFA); longterm survival outcome; multivariate analysis

Submitted Jun 18, 2021. Accepted for publication Oct 22, 2021.

doi: $10.21037 /$ tcr-21-1045

View this article at: https://dx.doi.org/10.21037/tcr-21-1045

\footnotetext{
$\wedge$ ORCID: 0000-0002-6440-7825.
} 


\section{Introduction}

Hepatocellular carcinoma (HCC) was ranked as the fifth most common malignancy and the second leading cause of cancer death worldwide (1). Liver transplantation and liver resection remain as the main curative treatments for early HCC. Liver resection can be performed as open or laparoscopic hepatectomy. With gaining evidence on its short-term benefits, which included shorter operative time $(2,3)$, decreased blood loss $(2,4)$, shorter hospital stay (2-6), and decreased overall morbidity (3-7), laparoscopic liver resection (LLR) had emerged as a valuable treatment option for selected patients (8). Recent publications had shown comparable oncological outcomes in terms of diseasefree survival and overall survival for LLR and open liver resection $(2-7,9)$. Nevertheless, only $30 \%$ of patients with HCC were able to undergo liver resection. Radiofrequency ablation (RFA) could achieve a radical cure effect and longterm survival similar to surgery for small HCCs (10-12). It also provided the advantages of minimal invasive treatment, more rapid recovery, and low morbidity and mortality $(13,14)$. However, these advantages were largely identified in studies comparing RFA with open hepatectomy. To date, there was no robust clinical trial comparing the efficacy of LLR and RFA especially for small HCC. This study aimed to compare the short-term and long-term outcomes of LLR and RFA for patients with small HCC using a propensity score matching analysis to minimize potential selection bias. Factors affecting survival were then identified with multivariate analysis. We present the following article in accordance with the STROBE reporting checklist (available at https://tcr.amegroups.com/ article/view/10.21037/tcr-21-1045/rc).

\section{Methods}

This was a single-center retrospective analysis of a prospectively collected database. Diagnosis of HCC was made based on contrast enhancement on cross-sectional imaging such as triphasic computed tomography (CT scan) or magnetic resonance imaging (MRI). All patients underwent RFA or LLR for small HCC (defined as BCLC stage 0 or A, sized $\leq 3 \mathrm{~cm}, \leq 3$ nodules on contrast CT scan or MRI with no evidence of macrovascular invasion) from April 2005 to August 2020 were included. All patients followed the same protocol of preoperative workup and investigations including blood tests to determine liver function and alpha fetoprotein (AFP) level, as well as radiological assessment using contrasts CT scan and/or
MRI. Tumors located in segment 7 or 8 were defined as posterosuperior lesions.

The selection criteria and operative technique for LLR in our center were described previously (15). In general, liver resection was first considered in all cases. RFA were generally performed for patients with small deep-seated intraparenchymal tumors contraindicated to major liver resections. Patients' preference was considered if the lesion was amenable to both treatments.

For LLR, a $12-\mathrm{mm}$ camera trocar was inserted at the subumbilical region, and 4-5 trocars of diameter 5-12 mm were used by the surgeon and assistant. Ultrasonic shear device or Cavitron Ultrasonic Surgical Aspirator (CUSA) was used to conduct liver parenchymal transection. Pringle maneuvers were employed selectively. Anatomical liver resection was defined as removal of the whole hepatic segment or subsegments supplied by the tumor bearing tributaries.

RFA were performed percutaneously using the Cooltip $^{\mathrm{TM}}$ RF Ablation System (Medtronic, USA) under local anesthesia. The RFA electrode was inserted under noncontrast ultrasound or CT scan guidance, with an intended ablative margin of at least $1 \mathrm{~cm}$. Drain was placed only when clinically indicated. All LLR and RFA were performed by the same team of hepatobiliary surgeons and interventional radiologists respectively.

Conversion from laparoscopic to open surgery, blood transfusion, morbidity, mortality, and duration of hospital stay were all recorded as procedure-related factors. R0 resection was defined as resection margin more than or equal to $1 \mathrm{~mm}$. Data on post-operative complications were classified according to the Clavien-Dindo Grading (16). Both groups of patients followed the same post-treatment surveillance protocol (15). In general, 6-monthly triphasic contrast CT scans and chest X-rays were performed. Additional contrast CT scans were performed at first and third month after RFA to detect any early local recurrence. Liver function tests and serum alpha-fetoprotein levels were assessed 3-monthly for the first two years, then 6-monthly. The date of recurrence was defined as the date of radiological recurrence. The development of new HCC foci in contact with a tumor that had been excised or ablated was characterized as local recurrence. A new lesion developing at a distance from the initial location was characterized as a new recurrence. Re-resection, microwave or RFA, transarterial chemoembolization (TACE), or systemic therapy were administered when needed.

1:3 propensity score matching was conducted to match 


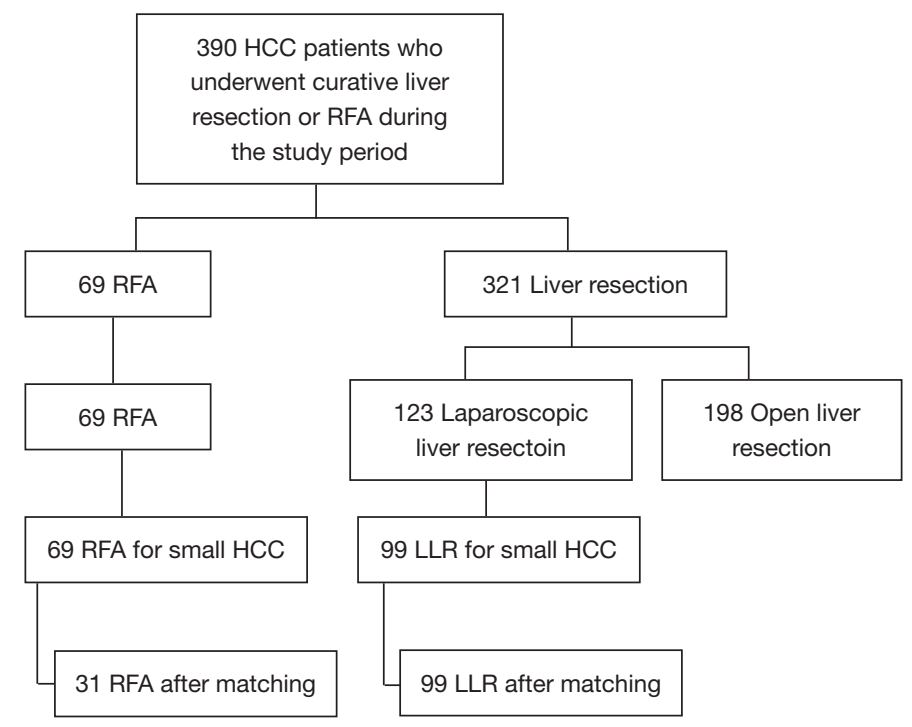

Figure 1 Flow diagram of participants at each stage of study.

patients in the LLR group and RFA group. Prognostic indicators, i.e., age, gender, tumor size, tumor number, Child's grading, albumin, bilirubin, platelet count, international normalized ratio, alpha-fetoprotein level and presence of cirrhosis on imaging were chosen for propensity score calculation. In our investigation, we used the genetic matching approach, which automatically adjusted the covariate balance between the two groups $(17,18)$. The two groups' demographics, tumor features, surgical data, postoperative results, and survival data were compared. The analysis was carried out on an intention-to-treat basis.

The study was conducted in accordance with the Declaration of Helsinki (as revised in 2013). The study was approved by The Hong Kong Hospital Authority Research Ethics Committee (Kowloon Central/Kowloon East) (The Hospital Authority manages 43 public hospitals and institutions in Hong Kong including Kwong Wah Hospital, No.: KC/KE-21-0241/ER-2) and informed consent was not required for retrospective study of a database.

\section{Statistical analysis}

Statistical analysis was performed with SPSS version 20 (SPSS Inc., Chicago, IL, USA). Continuous variables are expressed as mean \pm standard deviation (SD) or median with interquartile range (IQR) if the data is skewed. Student $t$-test or Mann-Whitney $U$ test were used to analyze continuous variables as appropriate, and Chi-square test was used for categorical variables. Survival was analyzed by Kaplan-
Meier method and compared using the log rank test. Statistical significance was set at $P$ value $\leq 0.05$. To identify variables related with survival, a multivariate analysis based on Cox regression was employed. The multivariate regression model includes all variables having a $\mathrm{P}$ value of 0.1 in the univariate analysis.

\section{Results}

During the study period, 99 and 69 patients underwent LLR and RFA for small HCC, respectively. After propensity score matching, there were 99 patients in the LLR group and 31 patients in the RFA group (Figure 1). All LLR were performed without hand-port or robotic assistance and all RFA were performed percutaneously; $45.5 \%$ of LLR were anatomical liver resection and Pringle maneuver was used in $3.0 \%$. The demographic and clinicopathological data were depicted in Table 1. There was no difference in the baseline characteristics including age, gender, hepatitis carrier status, cirrhosis, liver function, AFP level, tumor size and tumor number between the two groups. There were more tumors in the posterosuperior segments in RFA group $(58.1 \%$ vs. $41.9 \%, \mathrm{P}=0.002)$.

Regarding short-term post-operative outcomes, no significant difference was seen in blood transfusion, overall and major complication rates and 30-day mortality (Table 2). One patient in LLR group had laparoscopic segment 5 segmentectomy performed and complicated with gallbladder perforation required emergency laparoscopic 
Table 1 Demographic and clinicopathological data

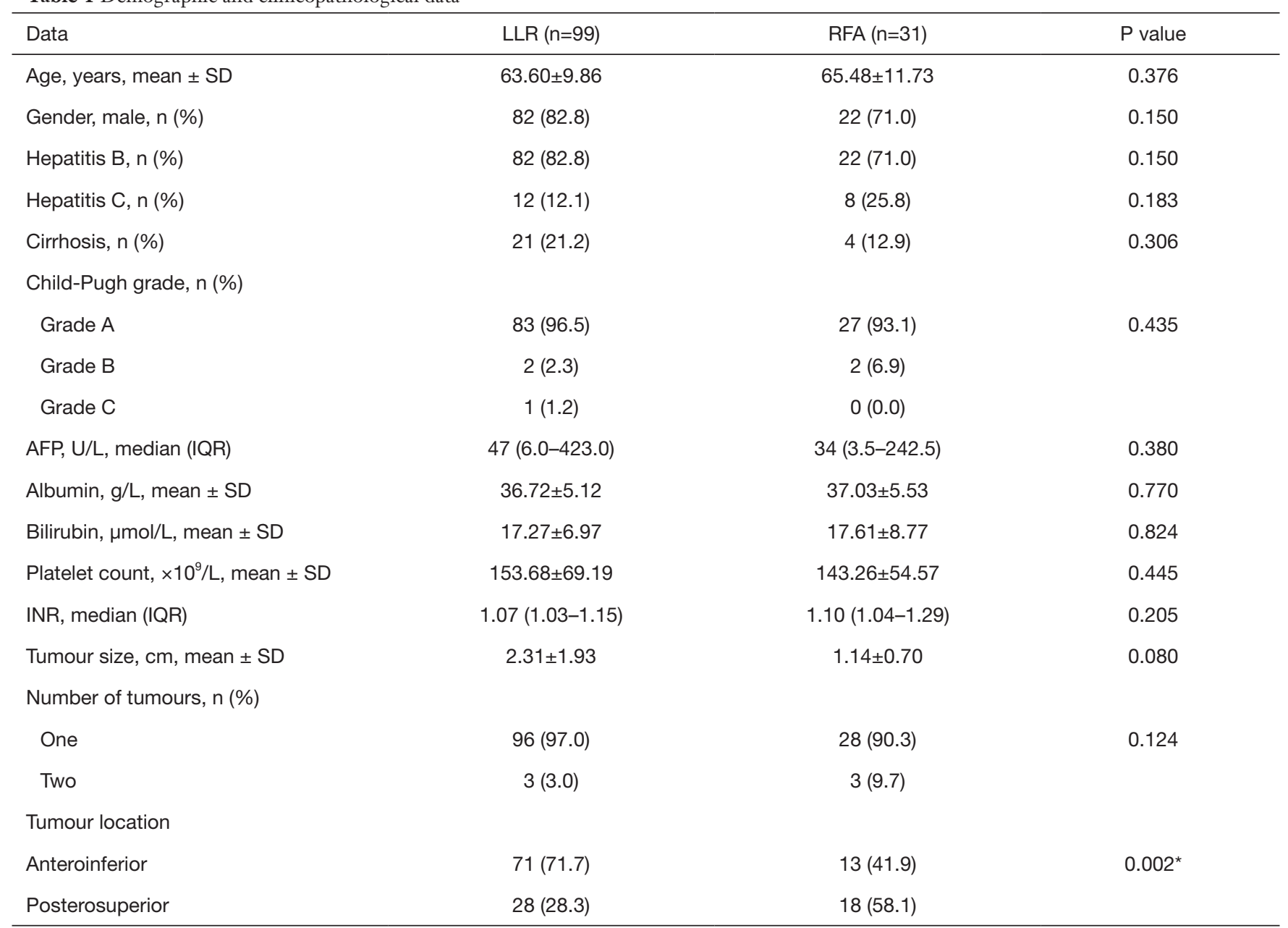

*, statistical significant. LLR, laparoscopic liver resection; RFA, radiofrequency ablation; SD, standard deviation; IQR, inter-quartile range; AFP, alpha-fetoprotein.

cholecystectomy. Another patient in LLR group died of hospital-acquired pneumonia 12 days after operation. Hospital stay was significantly shorter for the RFA group (3.06 vs. 6.05 days, $\mathrm{P}=0.001$ ).

Median follow-up was 34 months (range, 1-175 months). There were no lost to follow-up or missing data. The overall survival, disease-free survival and local recurrencefree survival were illustrated in Figures 2-4. The LLR group and RFA group had similar overall survival rate $(91.8 \%$ vs. $79.2 \%$ at 5 -year, $\mathrm{P}=0.060)$; while the LLR group had a significantly better disease-free survival rate $(49.0 \% \mathrm{vs}$. $30.3 \%$ at 5 -year, $\mathrm{P}=0.002)$ and local recurrence-free rate ( $96.0 \%$ vs. $63.7 \%$ at 5 -year, $\mathrm{P}<0.001$ ) when compared with the RFA group (Table 3).

Multivariate analysis showed that treatment received by patient (LLR vs. RFA), prothrombin time and platelet counts were significantly associated with disease-free survival. On the other hand, the only factor associated with local recurrence-free survival was the treatment received by patient (Tables 4,5).

\section{Discussion}

The selection of LLR versus RFA as the first line treatment for small HCC remains controversial. This study showed that both procedures were safe and feasible. RFA had a shorter hospital stay, while LLR had a lower local recurrence rate and better disease-free survival rate. Nevertheless, the overall survival was comparable between the two groups. 
Table 2 Short-term outcomes

\begin{tabular}{|c|c|c|c|}
\hline Outcomes & $\operatorname{LLR}(n=99)$ & RFA $(n=31)$ & $P$ value \\
\hline Anatomical resection, $\mathrm{n}(\%)$ & $45(45.5)$ & & \\
\hline Conversion, n (\%) & $6(6.1)$ & & \\
\hline Resection margin, mm, median (IQR) & $8[3-10]$ & & \\
\hline Transfusion, n (\%) & $12(12.1)$ & $1(3.2)$ & 0.150 \\
\hline All complications, $\mathrm{n}(\%)$ & $12(12.1)$ & $2(6.5)$ & 0.374 \\
\hline Major (grade 3 or above) complications, $\mathrm{n}(\%)$ & $1(1.0)$ & $0(0.0)$ & 0.574 \\
\hline 30-days mortality, n (\%) & $1(1.0)$ & $0(0.0)$ & 0.574 \\
\hline
\end{tabular}

*, statistical significant. LLR, laparoscopic liver resection; RFA, radiofrequency ablation; SD, standard deviation; IQR, inter-quartile range.

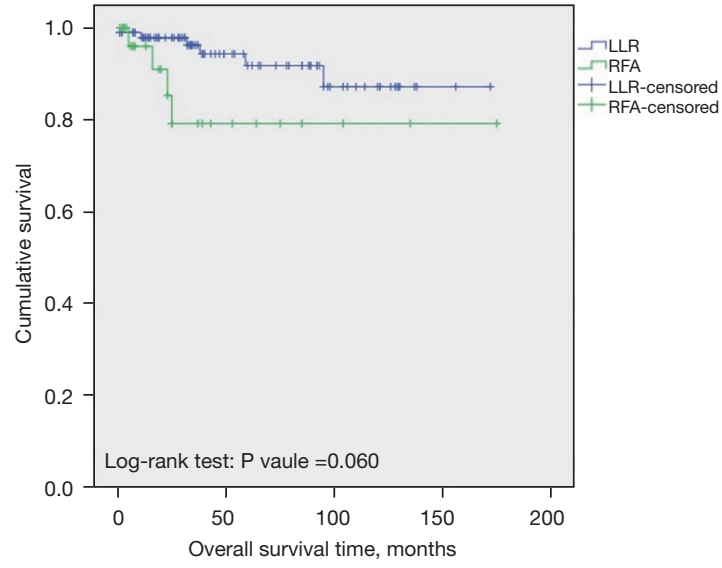

Figure 2 Kaplan-Meier curves for overall survival.

Two comparative studies on small HCC were published in 2020. Chong et al. performed a propensity score matching comparing 59 pairs of patients with BCLC stage 0/A HCC, who received minimally invasive treatment (19). $50.8 \%$ of LLR were performed with robotic assistance and only $84.5 \%$ of RFA were performed percutaneously. They found that the overall complication rate of the two groups was not significantly different $(5.1 \%$ vs. $1.7 \%, \mathrm{P}=0.625)$. LLR provided a significantly better 1-, 3-, and 5-year overall and disease-free survival (94.9\% vs. $96.6 \%, 88.2 \%$ vs. $78.7 \%, 82.5 \%$ vs. $53.3 \%, \mathrm{P}=0.005$ and $86.3 \%$ vs. $59.3 \%$, $68.0 \%$ vs. $25.3 \%, 68.0 \%$ vs. $15.9 \%, \mathrm{P}<0.001$ respectively), though there was no analysis on the recurrence pattern.

Another comparative study from Ogiso et al. compared

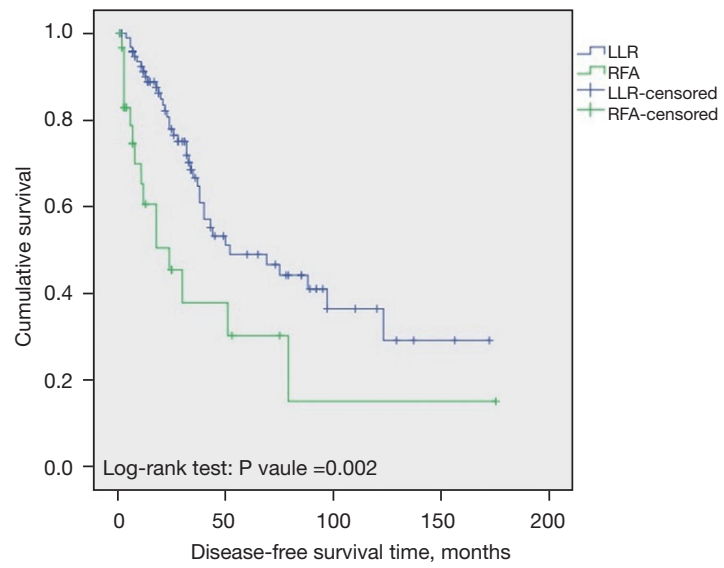

Figure 3 Kaplan-Meier curves for disease-free survival.

85 LLR with 136 RFA patients (20). They have the same definition of 'small HCC' as our center and we demonstrated similar results. Their result showed that LLR had a worse short-term outcome when compared with RFA in terms of more blood transfusion $(8.2 \% v s .0 \%, \mathrm{P}<0.001)$, more overall complications $(11.8 \%$ vs. $2.9 \%, \mathrm{P}=0.020)$ and longer hospital stay (11 days vs. 6 days, $\mathrm{P}<0.001)$. However, there were no significant difference in grade 3 or above complications between the two groups $(1.2 \%$ vs. $0 \%$, $\mathrm{P}=0.385)$. In spite of the minimally invasive nature of LLR, it still resulted in larger wounds than percutaneous RFA and it had to be performed under general anesthesia, thus resulting in longer hospital stay for post-operative recovery and pain control. 


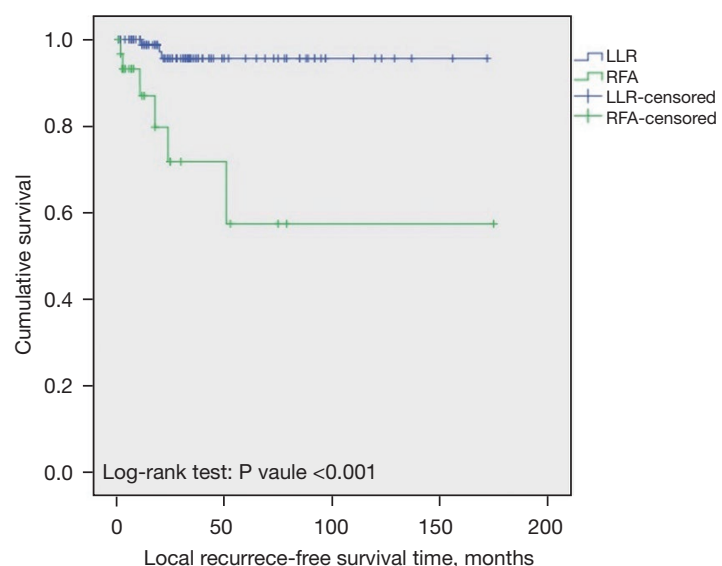

Figure 4 Kaplan-Meier curve for local recurrence-free survival.

Table 3 Long-term outcomes

\begin{tabular}{lccc}
\hline Outcomes & LLR $(\mathrm{n}=99)$ & $\mathrm{RFA}(\mathrm{n}=31)$ & $\mathrm{P}$ value \\
\hline Overall survival, \% & & & \\
1-year & 97.9 & 96.0 & 0.060 \\
3-year & 96.2 & 79.2 & \\
5-year & 91.8 & 79.2 & \\
Disease free survival, \% & & \\
1-year & 91.2 & 60.6 & $0.002^{*}$ \\
3-year & 66.7 & 37.9 & \\
5-year & 49.0 & 30.3 & \\
Local recurrence, & $3(3.0 \%)$ & $6(19.4 \%)$ & $0.002^{*}$ \\
n (\%) & & & \\
Local recurrence-free survival, \% & & \\
1-year & 98.8 & 88.3 & $<0.001^{*}$ \\
3-year & 96.0 & 76.4 & \\
5-year & 96.0 & 63.7 & \\
\hline
\end{tabular}

*, statistical significant. LLR, laparoscopic liver resection; RFA, radiofrequency ablation.

For long-term outcome, our study results concurred with previous studies and we showed that the benefit of disease-free survival and local recurrence-free survival for LLR extended to 5 -year post-treatment. The only factor associated with local recurrence is RFA on multivariate analysis. To date, there was no randomized controlled trial comparing the outcomes of LLR and RFA for small HCC. The meta-analysis by Li et al. showed that RFA group had
Table 4 Univariate analysis of survival

\begin{tabular}{lcc}
\hline \multirow{2}{*}{ Factors } & \multicolumn{2}{c}{ P value for } \\
\cline { 2 - 3 } & $\begin{array}{c}\text { Disease-free } \\
\text { survival }\end{array}$ & $\begin{array}{c}\text { Local recurrence-free } \\
\text { survival }\end{array}$ \\
\hline LLR vs. RFA & 0.004 & 0.002 \\
Age & 0.861 & 0.362 \\
Gender & 0.528 & 0.374 \\
Child's grade & 0.448 & 0.977 \\
Albumin & 0.132 & 0.621 \\
Bilirubin & 0.476 & 0.912 \\
PT & 0.004 & 0.109 \\
Platelet count & 0.006 & 0.199 \\
AFP & 0.765 & 0.589 \\
Number of lesions & 0.053 & 0.752 \\
Tumor size & 0.219 & 0.183 \\
\hline
\end{tabular}

LLR, laparoscopic liver resection; RFA, radiofrequency ablation; PT, prothrombin time; AFP, alpha-fetoprotein.

Table 5 Multivariate analysis of survival

\begin{tabular}{lccc}
\hline Survival and factors & Hazard ratio & $95 \% \mathrm{Cl}$ & P value \\
\hline Disease-free survival & & & \\
LLR vs. RFA & 1.929 & 1.045 to 3.562 & 0.036 \\
PT & 1.028 & 1.009 to 1.047 & 0.003 \\
Platelet count & 0.993 & 0.988 to 0.998 & 0.004 \\
Local recurrence-free survival & & \\
\multicolumn{2}{l}{$\begin{array}{l}\text { LLR vs. RFA } \\
\text { LLR, laparoscopic liver resection; RFA, radiofrequency ablation; }\end{array}$} \\
$\begin{array}{l}\text { PT, prothrombin time. } \\
\text { 9.247 }\end{array}$
\end{tabular}

lower complication rate, while LLR had a significantly better 1 - and 3-year disease-free survival and 5-year overall survival rate than the RFA group (21). However, the 8 retrospective studies included in the meta-analysis had different definition of the 'small HCC'. The most commonly used definitions were either the BCLC stage 0/A or the Milan criteria, yet the tumor size ranged from $<3$ to $<6.5 \mathrm{~cm}$. This meta-analysis showed that LLR had a significantly better 5 -year overall survival and better 1 - and 3 -year disease-free survival than RFA. Tumor size is one of the important factors in considering ablative treatment 
as the efficacy in complete ablation diminished with larger tumour size and local tumour progression was more frequent in larger tumours (22).

In order to achieve comparable long-term outcomes between RFA and LLR, careful patient selection is of utmost importance. HCC has the propensity to invade into the portal and hepatic veins, leading to intrahepatic metastasis, which are two factors proven to be associated with poor prognosis (23-30). With the systematic removal of liver parenchyma supplied by the tumor bearing tributaries $(31,32)$, anatomical liver resection was shown to have significantly better recurrence-free survival $(24,33)$. On the other hand, non-anatomical resection and RFA may leave behind non-perfused ischemic liver tissues which is known to be associated with early recurrence and poor survival after resection of HCC (34). In our series, $45.5 \%$ of our LLR were anatomical liver resection.

Furthermore, our multivariate analysis showed that disease-free survival was associated with prothrombin time and platelet counts. These two factors were indicative of the degree of underlying cirrhosis and advanced cirrhosis was an important factor contributing to recurrence after treatment for HCC (35-37). Though LLR provided a better local control, less than $30 \%$ of the patients with HCC could undergo resection (38). These patients have underlying cirrhosis and limited liver function reserve. For patients with marginal liver function or small deeply located tumors, RFA could serve as an alternative treatment modality with similar overall survival rate. The higher locoregional recurrence rate did not adversely affect the overall survival as the recurrence could be further treated with re-ablation or resection $(39,40)$.

Our study was limited by its retrospective design and a relatively small sample size. Only patients with small HCC who had been treated with pure LLR or percutaneous RFA were included. Despite the use of propensity score matching to ensure both groups were comparable and represented a homogenous and specific group of patients, there could be some unknown confounding factors that remain unrecognized in this retrospective study (41). Early post ablation (within 24 hours) contrast CT scan was not performed in our center to evaluate completeness of RFA ablation due to limited resources. In fact, only 1 patient $(3.2 \%)$ in our series suffered from recurrence within first month after RFA. Our incomplete ablation rate was similar to other published series $(19,42,43)$.

\section{Conclusions}

In conclusion, both RFA and LLR are safe and feasible treatment options for patients with small HCC. LLR should be considered for patients with preserved liver function with a better disease-free survival; while RFA offered a comparable overall survival with less surgical trauma and shorter hospital stay.

\section{Acknowledgments}

Funding: None.

\section{Footnote}

Reporting Checklist: The authors have completed the STROBE reporting checklist. Available at https://tcr. amegroups.com/article/view/10.21037/tcr-21-1045/rc

Data Sharing Statement: Available at https://tcr.amegroups. com/article/view/10.21037/tcr-21-1045/dss

Conflicts of Interest: Both authors have completed the ICMJE uniform disclosure form (available at https://tcr.amegroups. com/article/view/10.21037/tcr-21-1045/coif). The authors have no conflicts of interest to declare.

Ethical Statement: The authors are accountable for all aspects of the work in ensuring that questions related to the accuracy or integrity of any part of the work are appropriately investigated and resolved. The study was conducted in accordance with the Declaration of Helsinki (as revised in 2013). The study was approved by the Hong Kong Hospital Authority Research Ethics Committee (Kowloon Central/Kowloon East) (The Hospital Authority manages 43 public hospitals and institutions in Hong Kong including Kwong Wah Hospital, No.: KC/KE-210241/ER-2) and informed consent was not required for retrospective study of a database.

Open Access Statement: This is an Open Access article distributed in accordance with the Creative Commons Attribution-NonCommercial-NoDerivs 4.0 International License (CC BY-NC-ND 4.0), which permits the noncommercial replication and distribution of the article with the strict proviso that no changes or edits are made and the original work is properly cited (including links to both the 
formal publication through the relevant DOI and the license). See: https://creativecommons.org/licenses/by-nc-nd/4.0/.

\section{References}

1. Ferlay J, Soerjomataram I, Dikshit R, et al. Cancer incidence and mortality worldwide: sources, methods and major patterns in GLOBOCAN 2012. Int J Cancer 2015;136:E359-86.

2. Cheung TT, Dai WC, Tsang SH, et al. Pure Laparoscopic Hepatectomy Versus Open Hepatectomy for Hepatocellular Carcinoma in 110 Patients With Liver Cirrhosis: A Propensity Analysis at a Single Center. Ann Surg 2016;264:612-20.

3. Memeo R, de'Angelis N, Compagnon P, et al. Laparoscopic vs. open liver resection for hepatocellular carcinoma of cirrhotic liver: a case-control study. World J Surg 2014;38:2919-26.

4. Takahara T, Wakabayashi G, Beppu T, et al. Longterm and perioperative outcomes of laparoscopic versus open liver resection for hepatocellular carcinoma with propensity score matching: a multi-institutional Japanese study. J Hepatobiliary Pancreat Sci 2015;22:721-7.

5. Xiang L, Li J, Chen J, et al. Prospective cohort study of laparoscopic and open hepatectomy for hepatocellular carcinoma. Br J Surg 2016;103:1895-901.

6. Sposito C, Battiston C, Facciorusso A, et al. Propensity score analysis of outcomes following laparoscopic or open liver resection for hepatocellular carcinoma. Br J Surg 2016;103:871-80.

7. Komatsu S, Brustia R, Goumard C, et al. Laparoscopic versus open major hepatectomy for hepatocellular carcinoma: a matched pair analysis. Surg Endosc 2016;30:1965-74.

8. Wakabayashi G, Cherqui D, Geller DA, et al. Recommendations for laparoscopic liver resection: a report from the second international consensus conference held in Morioka. Ann Surg 2015;261:619-29.

9. Ho KM, Cheng KC, Chan FK, et al. Laparoscopic hepatectomy versus open hepatectomy for hepatocellular carcinoma: A propensity case-matched analysis of the long-term survival. Ann Hepatobiliary Pancreat Surg 2021;25:1-7.

10. Kan X, Wang Y, Han P, et al. Combined ultrasound/ computed tomography guidance in percutaneous radiofrequency ablation after transarterial chemoembolization for hepatocellular carcinoma in the hepatic dome. Cancer Manag Res 2019;11:7751-7.
11. Lai C, Jin RA, Liang X, et al. Comparison of laparoscopic hepatectomy, percutaneous radiofrequency ablation and open hepatectomy in the treatment of small hepatocellular carcinoma. J Zhejiang Univ Sci B 2016;17:236-46.

12. Liu Z, Zhou Y, Zhang P, et al. Meta-analysis of the therapeutic effect of hepatectomy versus radiofrequency ablation for the treatment of hepatocellular carcinoma. Surg Laparosc Endosc Percutan Tech 2010;20:130-40.

13. Wong J, Lee KF, Yu SC, et al. Percutaneous radiofrequency ablation versus surgical radiofrequency ablation for malignant liver tumours: the long-term results. HPB (Oxford) 2013;15:595-601.

14. Ng KK, Poon RT, Lam CM, et al. Efficacy and safety of radiofrequency ablation for perivascular hepatocellular carcinoma without hepatic inflow occlusion. Br J Surg 2006;93:440-7.

15. Chan FK, Cheng KC, Yeung YP. Laparoscopic liver resection: lessons learnt after 100 cases. Hong Kong Med J 2014;20:386-92.

16. Dindo D, Demartines N, Clavien PA. Classification of surgical complications: a new proposal with evaluation in a cohort of 6336 patients and results of a survey. Ann Surg 2004;240:205-13.

17. Brookhart MA, Schneeweiss S, Rothman KJ, et al. Variable selection for propensity score models. Am J Epidemiol 2006;163:1149-56.

18. Diamond A, Sekhon JS. Genetic matching for estimating causal effects: a general multivariate matching method for achieving balance in observational studies. Rev Econ Stat 2013;95:932-45.

19. Chong CC, Lee KF, Chu CM, et al. Laparoscopic Hepatectomy (with or without Robotic Assistance) versus Radiofrequency Ablation as a Minimally Invasive Treatment for Very Early-Stage or Early-Stage Hepatocellular Carcinoma. Dig Surg 2020;37:65-71.

20. Ogiso S, Seo S, Eso Y, et al. Laparoscopic liver resection versus percutaneous radiofrequency ablation for small hepatocellular carcinoma. HPB (Oxford) 2021;23:533-7.

21. Li X, Wu YS, Chen D, et al. Laparoscopic hepatectomy versus radiofrequency ablation for hepatocellular carcinoma: a systematic review and meta-analysis. Cancer Manag Res 2019;11:5711-24.

22. Di Costanzo GG, Tortora R, Opramolla A, et al. Thermal ablation of large unresectable hepatocellular carcionoma in cirrhotic patients. Hepatoma Res 2018;4:53.

23. Yamanaka N, Okamoto E, Toyosaka A, et al. Prognostic factors after hepatectomy for hepatocellular carcinomas. A univariate and multivariate analysis. Cancer 
1990;65:1104-10.

24. Franco D, Capussotti L, Smadja C, et al. Resection of hepatocellular carcinomas. Results in 72 European patients with cirrhosis. Gastroenterology 1990;98:733-8.

25. Chen MF, Hwang TL, Jeng LB, et al. Hepatic resection in 120 patients with hepatocellular carcinoma. Arch Surg 1989;124:1025-8.

26. Jwo SC, Chiu JH, Chau GY, et al. Risk factors linked to tumor recurrence of human hepatocellular carcinoma after hepatic resection. Hepatology 1992;16:1367-71.

27. Kosuge T, Makuuchi M, Takayama T, et al. Longterm results after resection of hepatocellular carcinoma: experience of 480 cases. Hepatogastroenterology 1993;40:328-32.

28. Vauthey JN, Klimstra D, Franceschi D, et al. Factors affecting long-term outcome after hepatic resection for hepatocellular carcinoma. Am J Surg 1995;169:28-34; discussion 34-5.

29. Shimada M, Takenaka K, Fujiwara Y, et al. Des-gammacarboxy prothrombin and alpha-fetoprotein positive status as a new prognostic indicator after hepatic resection for hepatocellular carcinoma. Cancer 1996;78:2094-100.

30. Izumi R, Shimizu K, Ii T, et al. Prognostic factors of hepatocellular carcinoma in patients undergoing hepatic resection. Gastroenterology 1994;106:720-7.

31. Makuuchi M, Hasegawa H, Yamazaki S. Ultrasonically guided subsegmentectomy. Surg Gynecol Obstet 1985;161:346-50.

32. Cheng KC, Ho KM. Anatomical laparoscopic right posterior sectionectomy. Ann Laparosc Endosc Surg 2017;2:36.

33. Imamura $H$, Matsuyama $Y$, Miyagawa $Y$, et al. Prognostic significance of anatomical resection and des-gammacarboxy prothrombin in patients with hepatocellular carcinoma. Br J Surg 1999;86:1032-8.

34. Cho JY, Han HS, Choi Y, et al. Association of Remnant

Cite this article as: Cheng KC, Ho KM. Pure laparoscopic liver resection versus percutaneous radiofrequency ablation for small hepatocellular carcinoma: a propensity score and multivariate analysis. Transl Cancer Res 2022;11(1):43-51. doi: $10.21037 /$ tcr-21-1045
Liver Ischemia With Early Recurrence and Poor Survival After Liver Resection in Patients With Hepatocellular Carcinoma. JAMA Surg 2017;152:386-92.

35. Sasaki Y, Imaoka S, Masutani S, et al. Influence of coexisting cirrhosis on long-term prognosis after surgery in patients with hepatocellular carcinoma. Surgery 1992;112:515-21.

36. Tung-Ping Poon R, Fan ST, Wong J. Risk factors, prevention, and management of postoperative recurrence after resection of hepatocellular carcinoma. Ann Surg 2000;232:10-24.

37. Guglielmi A, Ruzzenente A, Sandri M, et al. Radio frequency ablation for hepatocellular carcinoma in cirrhotic patients: prognostic factors for survival. J Gastrointest Surg 2007;11:143-9.

38. Tang ZY. Hepatocellular carcinoma surgery--review of the past and prospects for the 21 st century. J Surg Oncol 2005;91:95-6.

39. Imai K, Beppu T, Chikamoto A, et al. Salvage treatment for local recurrence of hepatocellular carcinoma after local ablation therapy. Hepatol Res 2014;44:E335-45.

40. Taura K, Ikai I, Hatano E, et al. Implication of frequent local ablation therapy for intrahepatic recurrence in prolonged survival of patients with hepatocellular carcinoma undergoing hepatic resection: an analysis of 610 patients over 16 years old. Ann Surg 2006;244:265-73.

41. Talari K, Goyal M. Retrospective studies - utility and caveats. J R Coll Physicians Edinb 2020;50:398-402.

42. Huang JW, Hernandez-Alejandro R, Croome KP, et al. Surgical vs percutaneous radiofrequency ablation for hepatocellular carcinoma in dangerous locations. World J Gastroenterol 2011;17:123-9.

43. Khan MR, Poon RT, Ng KK, et al. Comparison of percutaneous and surgical approaches for radiofrequency ablation of small and medium hepatocellular carcinoma. Arch Surg 2007;142:1136-43; discussion 1143. 